$9-7-2021$

\title{
Atención de la salud sexual y reproductiva de mujeres migrantes: Un mapeo de actores de la sociedad civil en Tabasco, México-2020
}

Silvana Larrea-Schiavon

Population Council

Lucía Vázquez Quesada

Population Council

Esperanza Basurto-Alcalde

Natalia Polgovsky

Isabel Vieitez Martínez

Population Council

See next page for additional authors

Follow this and additional works at: https://knowledgecommons.popcouncil.org/departments_sbsr-rh How does access to this work benefit you? Let us know!

\section{Recommended Citation}

Larrea-Schiavon, Silvana, Lucía Vázquez-Quesada, Esperanza Basurto-Alcalde, Natalia Polgovsky, Isabel Vieitez Martínez, and Pooja Sripad. 2021. "Atención de la salud sexual y reproductiva de mujeres migrantes: Un mapeo de actores de la sociedad civil en Tabasco, México-2020." México: Population Council. 


\section{Authors}

Silvana Larrea-Schiavon, Lucía Vázquez Quesada, Esperanza Basurto-Alcalde, Natalia Polgovsky, Isabel Vieitez Martínez, and Pooja Sripad 


\section{Atención de la salud sexual y}

reproductiva de mujeres migrantes: Un mapeo de actores de la sociedad civil en Tabasco, México 2020

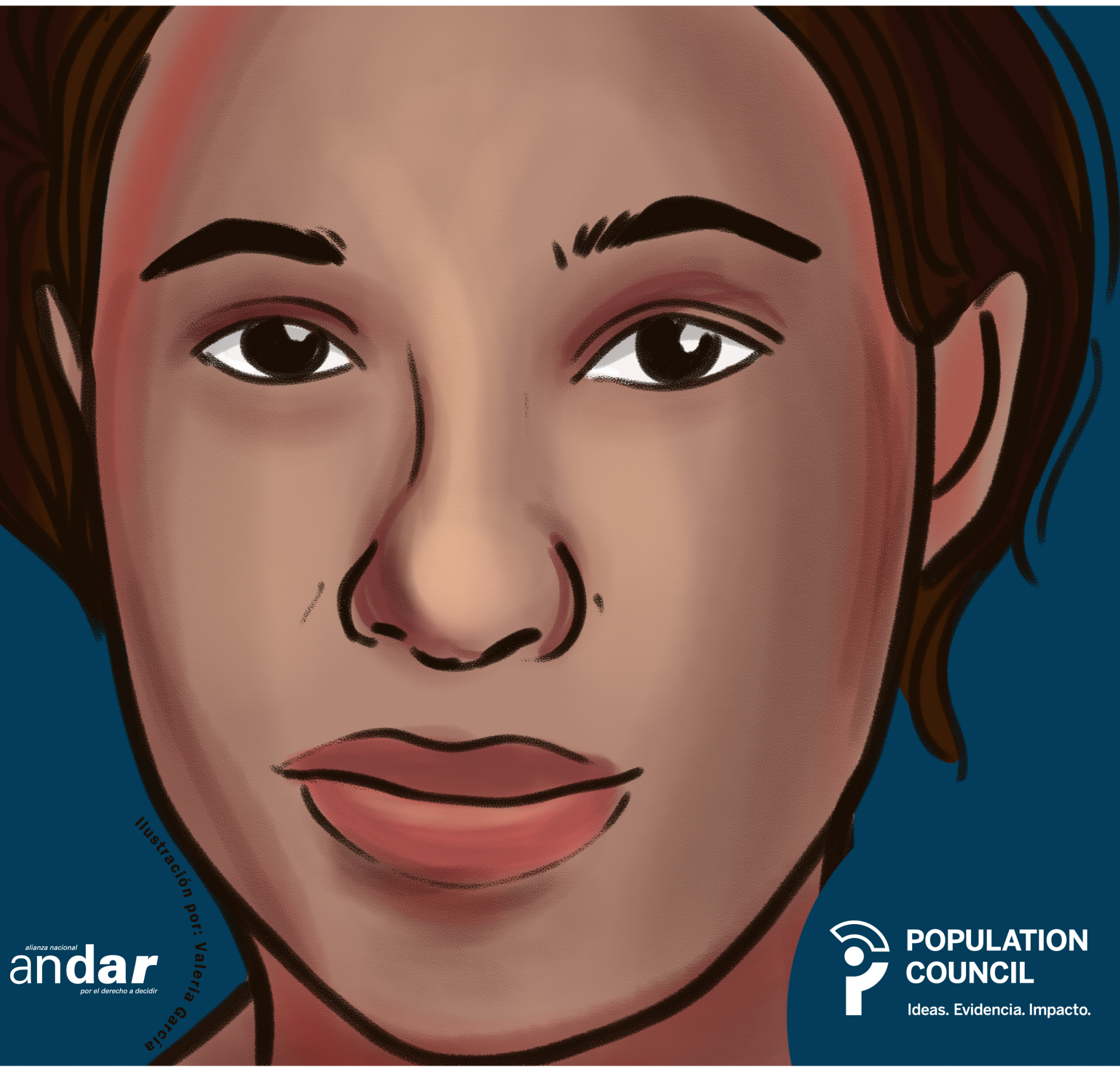




\title{
Population COUNCIL
}

Ideas. Evidencia. Impacto.

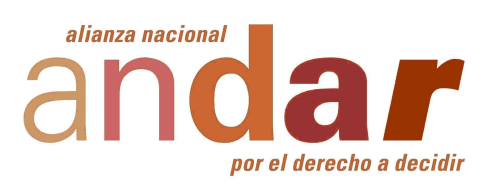

\begin{abstract}
Atención de la salud sexual y reproductiva de mujeres migrantes: Un mapeo de actores de la sociedad civil en Tabasco, México. 2020.
\end{abstract}

\section{Equipo de investigación:}

Protocolo de investigación, análisis de datos, redacción y/o revisión de informe:

Silvana Larrea-Schiavon* Lucía Vázquez-Quesada* Isabel Vieitez Martínez*

Pooja Sripad*

*Population Council.

Coordinación del proyecto, recolección y análisis de datos, redacción y revisión de informe:

Esperanza Basurto-Alcalde, consultora.

Recolección de datos, análisis de datos, y redacción de informe:

Natalia Polgovsky-Ezcurra, consultora.

Recolección de datos (personas pasantes y voluntarias):

Arturo Alamilla, entrevistador. Ana Regina Nájera, entrevistadora. Ximena Nateras, entrevistadora. Iván Coria, entrevistador. Gemma Hernández, entrevistadora.

\section{Corrección de estilo:}

Claudia Martínez.

\section{Ilustración y diseño editorial:}

Valeria García.

\section{Agradecimientos:}

Agradecemos el esfuerzo realizado por el equipo de investigación, sobre todo a personas pasantes y voluntarias, así como la gran disposición que las personas entrevistadas tuvieron para compartirnos su experiencia, en pleno inicio de la pandemia causada por el virus SARS-CoV-2, y por su compromiso constante con las mujeres migrantes y otras poblaciones en movilidad.

\section{Aviso legal:}

Se permite la reproducción total o parcial de esta publicación para fines educativos, de investigación y cualquier otro fin libre de lucro, siempre y cuando se cite la fuente.

\section{Cita sugerida:}

Larrea-Schiavon Silvana, Vázquez-Quesada Lucía, Basurto-Alcalde Esperanza, Polgovsky Natalia, Vieitez Isabel, and Sripad Pooja. 2021. "Atención de la salud sexual y reproductiva de mujeres migrantes: Un mapeo de actores de la sociedad civil en Tabasco, México. 2020." México: Population Council. 


\section{HALLAZGOS PRINCIPALES}

- Tabasco es un estado fronterizo con Guatemala, por el que atraviesan las rutas migratorias del sureste del país. Es la segunda entidad, solo después de Chiapas, con mayor número de solicitudes de asilo y refugio en el país.

- Tenosique es el municipio del estado en el que se concentra mayor número de población migrante.

- Las 9 organizaciones de la sociedad civil (OSCs) entrevistadas tienen características muy diversas, incluyen albergues, organizaciones locales y colectivas vinculadas a la salud sexual y reproductiva (SSyR), y organizaciones internacionales, incluyendo los diversos perfiles que dan atención a las mujeres migrantes en tránsito.

- Los tres temas con mayor intervención fueron los métodos anticonceptivos, las infecciones de transmisión sexual (ITS) y pruebas de embarazo. Los temas con menor intervención fueron interrupción del embarazo, anticoncepción de emergencia y atención del parto.

- El contexto de movilidad, junto con el desconocimiento del contexto y las normativas, así como los costos económicos, fueron factores destacados que dificultan la atención. Junto a ello, se identificó un gran número de barreras relacionadas directamente con instituciones de salud.

- La sensibilización del personal médico en torno a la SSyR de mujeres migrantes se considera fundamental para que mejore el servicio.

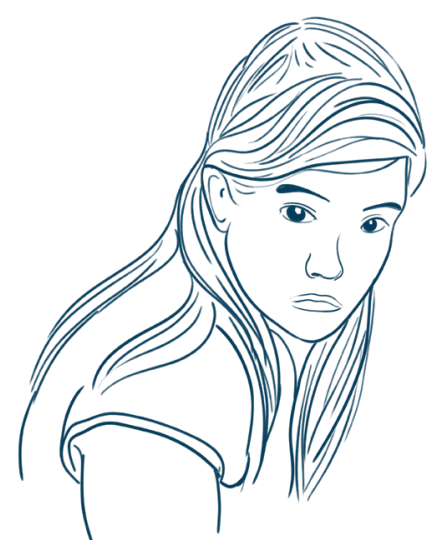

Nota de campo Son mujeres distintas, vienen de culturas distintas y diferentes países. -Organización, Tabasco

\section{INTRODUCCIÓN}

Tabasco comparte 290 kilómetros de frontera con Guatemala, lo que lo convierte en un estado de entrada y tránsito de personas migrantes. El principal punto de cruce por Guatemala a Tabasco es desde el municipio guatemalteco de El Naranjo. El Ceibo es el puerto fronterizo, mientras que El Pedregal y La Palma son pueblos a los que se accede por su cercanía al río San Pedro, frontera natural entre México y Guatemala. Estos últimos dos pueblos se ubican a unos $50 \mathrm{~km}$ aproximadamente de Tenosique y son parte de este municipio (Leyva-Flores et al., 2016).

De acuerdo con la Encuesta Sobre la Población Refugiada en México de 2017 (ENPORE), en el estado de Tabasco se registró un $3.6 \%$ de viviendas de personas refugiadas y un $33.8 \%$ de personas solicitantes de refugio. Esto quiere decir que Tabasco es la segunda entidad con mayor número de personas solicitantes de refugio en todo el país, solo por debajo del estado de Chiapas (INEGI, 2017). Con base en datos de la Unidad de Política Migratoria, Registro e Identidad de Personas (UPMRIP) de la Secretaría de Gobernación de México, de enero a julio de 2019 y 2020, 8.1\% de los eventos de personas extranjeras presentadas ante la autoridad migratoria mexicana sucedió en Tabasco, ocupando el tercer lugar en el país (UPMRIP, 2020).

La ruta del Golfo es considerada como la más peligrosas para transitar por México. Ésta cruza por tres estados: Tabasco, Veracruz y Tamaulipas. Informes publicados por instancias que abogan por los derechos humanos de las personas migrantes, así como la Encuesta sobre Agresión y Abuso al Migrante (EAAM) dan cuenta de un mayor número de agresiones y abusos contra personas migrantes en esta ruta, lo cual puede estar relacionado con la presencia de delincuencia organizada, principalmente los Zetas y el Cártel del Golfo (COLEF e INM, 2012). Por otro lado, con base en los indicadores publicados en el informe de la Red de Documentación de las Organizaciones Defensoras de Migrantes (REDODEM) 2019, Chiapas, Tabasco, Veracruz y Tamaulipas son los estados dónde se reportó el mayor porcentaje de agresiones físicas, privación de la libertad, privación de la vida y agresiones sexuales a migrantes (REDODEM, 2019).

El Eje Transversal no. 4 del Plan Estatal de Desarrollo 20192024 del estado de Tabasco, denominado Inclusión e Igualdad Sustantiva, incluye un capítulo sobre frontera sur y migración nacional e internacional. Este Eje transversal cuenta con dos programas prioritarios relacionados con migración y salud: Unidades móviles de auxilio en los puntos 
identificados de entrada de migrantes y Aplicar programas de salud reproductiva y seguimiento a enfermedades crónicodegenerativas como la diabetes o el VIH en municipios fronterizos y de paso de migrantes (Gobierno del Estado de Tabasco, 2019).

En relación con la salud sexual y reproductiva, en Tabasco el aborto es legal bajo tres causales: violación, riesgo para la vida de la mujer e inseminación artificial sin consentimiento. El aborto ilegal está tipificado como delito no grave, por lo que las mujeres pueden seguir el proceso penal en libertad (Andar, 2020). No obstante, muchas de las mujeres sujetas a proceso penal son de escasos recursos económicos. El Grupo de Información en Reproducción Elegida (GIRE) ha documentado que las fianzas impuestas van de los \$1,000 a los $\$ 197,000$ pesos mexicanos, lo que impide a muchas mujeres pagarla. La pena privativa de la libertad va de seis meses hasta tres años según el código vigente (GIRE, 2015).

\section{OBJETIVO Y METODOLOGÍA}

El presente estudio se enfocó en identificar si las organizaciones de la sociedad civil brindan los servicios de difusión de información, consejería, canalización, acompañamiento y atención directa a mujeres migrantes en tránsito por México en temas referentes a métodos anticonceptivos, anticoncepción de emergencia, interrupción del embarazo, pruebas de embarazo, atención prenatal, atención del parto, atención del postparto, salud menstrual, infecciones de transmisión sexual (ITS) y atención a sobrevivientes de violencia sexual.

Para alcanzar dicho objetivo se hizo una búsqueda dirigida de organizaciones que trabajaran en temas de salud sexual y reproductiva y/o con mujeres migrantes en siete estados claves en la ruta migratoria en la República mexicana: Chiapas, Tabasco, Veracruz, Oaxaca, Ciudad de México, Chihuahua y Baja California. Se identificó a un actor clave dentro de cada organización y se realizó una entrevista telefónica semiestructurada (para más información ver el reporte general del proyecto). En este documento se presentan los resultados para Tabasco.

\section{RESULTADOS}

\section{Perfil de las organizaciones entrevistadas}

Por búsqueda dirigida se identificó a un total de 14 organizaciones que cumplían con los criterios de inclusión, de las cuáles se logró entrevistar a 9 (64.2\%): 5 organizaciones locales y 4 internacionales, de las cuales 2 se auto identificaron como feministas y 3 eran albergues o casas para migrantes.

De las 9 organizaciones entrevistadas, 6 se encuentran en Tenosique, mientras que las otras 3 están localizadas en Villahermosa. Al ser Tenosique la ciudad con un mayor flujo de migrantes en el estado de Tabasco se podría intuir que hay una concentración más alta de organizaciones enfocadas en migración en dicha ciudad.

\section{Servicios brindados para la atención de la} SSyR de las mujeres migrantes

Del total de organizaciones entrevistadas en Tabasco, 5 (55.5\%) difunden información, 6 (66.6\%) dan consejería, 9 (100\%) canalizan casos, 8 (88.8\%) acompañan a las mujeres durante la atención y 1 (11.1\%) brinda atención directa en alguno de los temas mencionados.

Cuando se hace el cruce entre tipo de servicio (difusión de información, consejería, atención directa, canalización y referencia y acompañamiento) y temática de SSyR en la cual se ofertó este servicio, se identifica que los principales temas sobre los cuales se difundió información en las organizaciones fueron métodos anticonceptivos, (ITS) y pruebas de embarazo. Con respecto a los temas sobre los cuáles se brindó consejería, se identificaron métodos anticonceptivos e ITS. La atención directa brindada por las

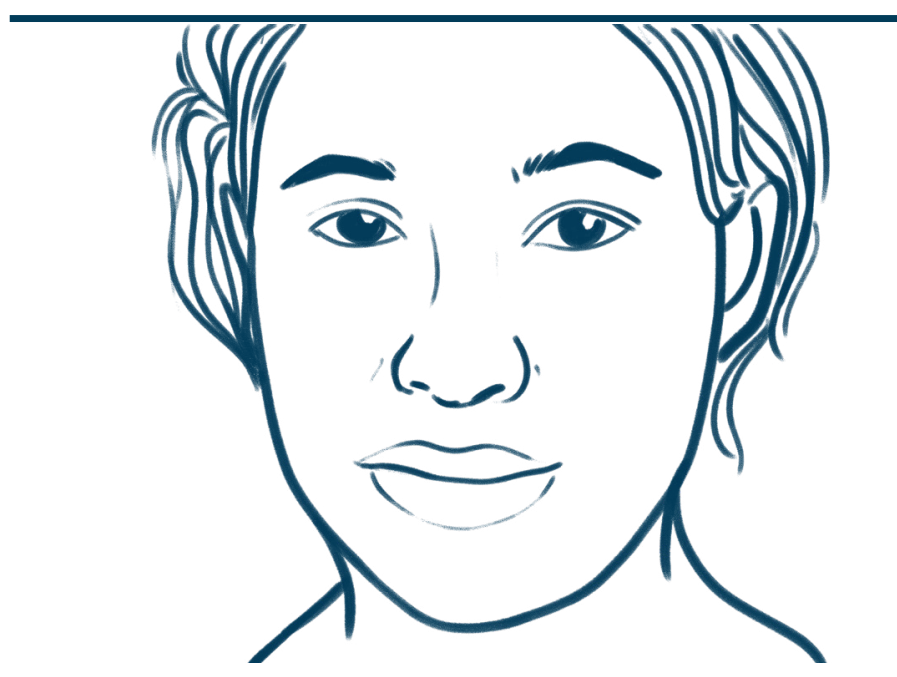

Nota de campo Se da la atención por el médico legista y se inicia el procedimiento legal sin consentimiento de la víctima. La denuncia puede demorar la estancia, la atención a una persona sobreviviente puede llevar 6 a 8 horas para que sea atendida, a veces varios días, son horas valiosas para acceder a un antiretroviral o una pastilla de anticoncepción de emergencia. Si ocurre un evento así en viernes por la tarde les dicen que vayan el lunes.d. - Organización, Tabasco 
organizaciones se concentró principalmente alrededor de métodos anticonceptivos. La canalización y referencia, así como el acompañamiento de casos, fueron brindados por más del $66.6 \%$ de las organizaciones para todas las temáticas preguntadas (excepto para interrupción del embarazo).

Es relevante mencionar que solamente 1 organización indicó estar capacitada para brindar atención directa para informar sobre la interrupción del embarazo. 3 osCs dijeron brindar anticoncepción de emergencia a las mujeres migrantes (el número más elevado en este servicio en los siete estados incluidos en el estudio). Con relación a la salud menstrual, las organizaciones señalaron que las toallas higiénicas y los tampones son los más eficientes para las mujeres migrantes. Las copas menstruales se descartan debido a las condiciones de poca higiene en la que viajan las usuarias, por lo cual no tienen acceso a los medios para lavarla adecuadamente. Las OSCs que brindan atención para ITS tienen acceso a antirretrovirales y otros medicamentos para tratar estas infecciones (ej. Ceftriaxona inyectada). Por último, la atención que se dio a las víctimas de violencia sexual incluyó terapia psicológica, canalización, acompañamiento integral (con

Tabla 1. Porcentaje de organizaciones que ofrecen servicios de salud sexual y reproductiva, por tipo de servicio ofertado. Tabasco, 2020.

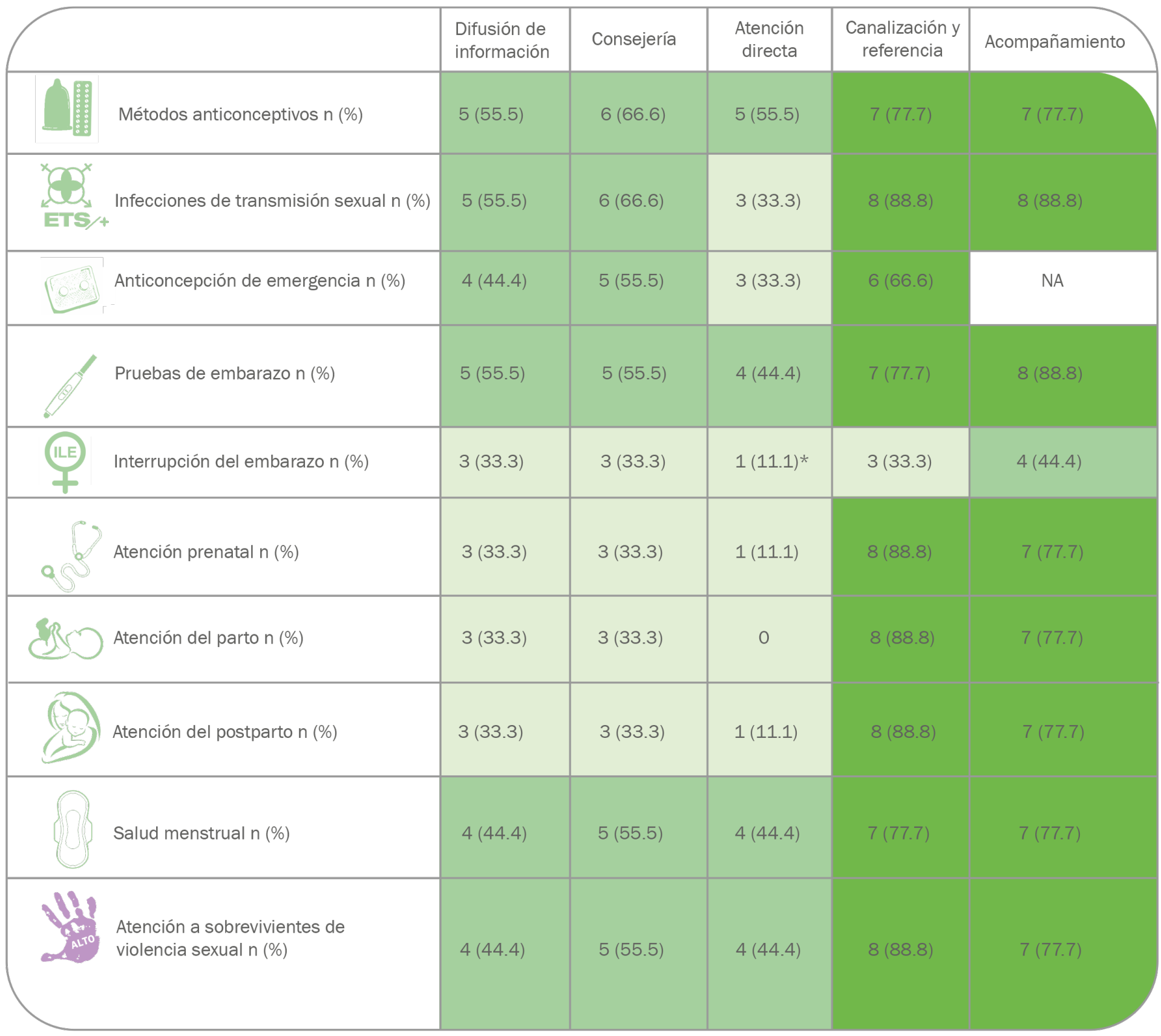

Nota: verde obscuro el servicio es ofertado por $>70 \%$ de las organizaciones entrevistadas; verde intermedio, el servicio es ofertado por entre $36 \%$ - $69 \%$ de las organizaciones entrevistadas; verde claro, el servicio es ofertado por $<36 \%$ de las organizaciones entrevistadas.

* La atención directa en el tema de interrupción del embarazo consiste en brindar información pública amplia sobre el marco legal (particularmente de la NOM-046), los métodos recomendados por la Organización Mundial de la Salud, y los requisitos para acceder a un servicio legal. Las OSCs no realizan los procedimientos, ni promueven la interrupción del embarazo - pues realizarlo es decisión de la mujer embarazada y de quien ella quiera involucrar al tomarla-. Se diferencia de "difusión de información" en tanto los temas cubiertos son más amplios en esta categoría. 
psicólogas, abogadas y personal médico) e inyección de ceftriaxona (ver Tabla1).

La posibilidad de que las organizaciones brinden atención directa depende, en cierta medida, de la presencia de personal de salud en sus instalaciones. Para Tabasco, 3 de las 9 organizaciones entrevistadas cuentan con personal médico en sus instalaciones; 1 con presencia cinco días a la semana, 1 cuatro días a la semana y 1 solamente un día a la semana (ver Gráfica 1).

Principales barreras para la atención en SSyR a mujeres migrantes

En las entrevistas con organizaciones de la sociedad civil en Tabasco se identificaron múltiples barreras para que las mujeres migrantes pudieran acceder a los servicios de SSyR durante su estancia o tránsito por el estado. En el caso del presente estudio, las barreras para el acceso a la salud fueron clasificadas en cuatro categorías:

1. Normativas: relacionadas con la legislación, políticas y normatividad que aplican tanto a la población migrante (marco restrictivo para la atención), como a las instituciones de salud;

2. Institucionales: impedimentos estructurales de la institución de salud que brinda o debería brindar los servicios;

3. Organizacionales: impedimentos estructurales de la organización de la sociedad civil que brinda los servicios; e

4. Individuales: obstáculos derivados de la condición socioeconómica, cultural y subjetiva de las personas migrantes (ver Tabla 2).

El mayor número de barreras identificadas por las organizaciones entrevistadas fueron las institucionales, es decir, las relacionadas directamente con las unidades de salud públicas y, en segundo lugar, las individuales. Para el caso de Tabasco, no se identificaron barreras normativas.

Las barreras institucionales identificadas, es decir, asociadas a las instituciones de salud, fueron 1) infraestructura insuficiente de las unidades de salud, ocasionando saturación de los servicios y tiempos de espera muy prolongados - la mayor parte de las unidades de salud presentes en los municipios donde se encuentran las organizaciones entrevistadas son unidades de primer nivel, lo cual dificulta el acceso a servicios de SSyR más especializados-; 2) las mujeres migrantes que requieren una atención más especializada se deben trasladar a otros
Gráfica 2. Porcentaje de organizaciones que cuentan con personal de salud en sus instalaciones. Tabasco, 2020. .

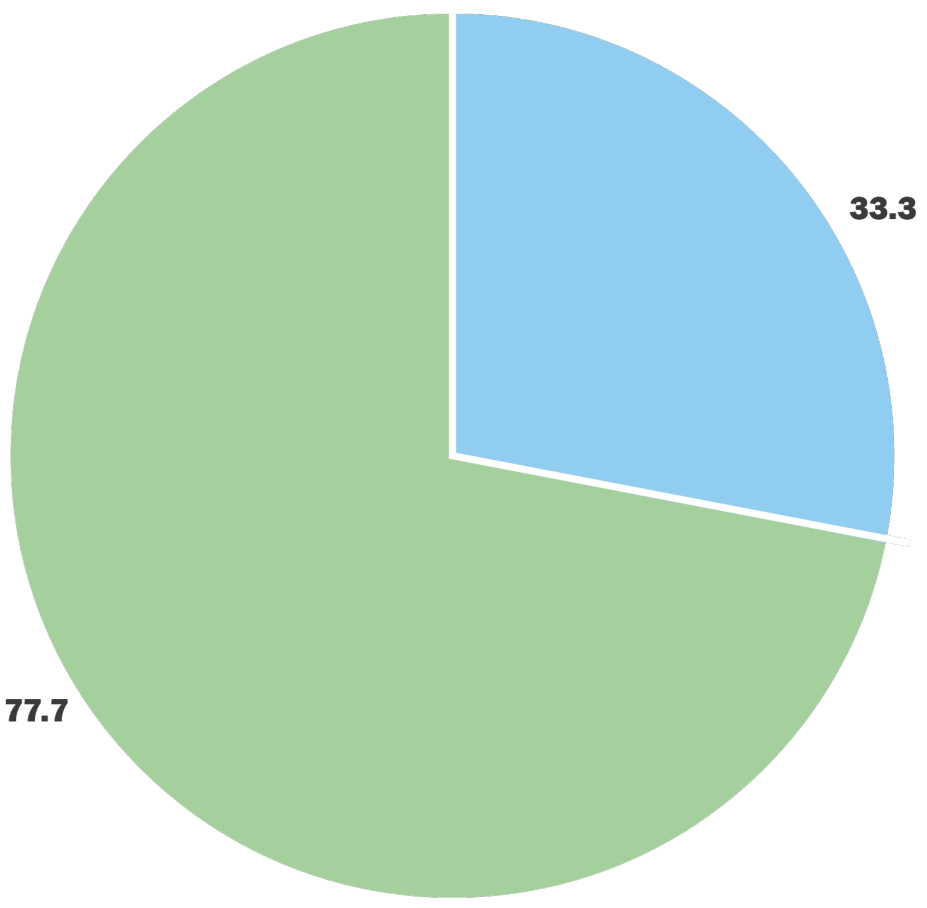

Con personal médico

Sin personal médico

municipios, sin embargo, el traslado se complica debido al costo de éste y a que las mujeres migrantes requieren de autorización para moverse de la ciudad; 3) la falta de insumos, principalmente relacionados con métodos anticonceptivos y reactivos para la detección de ITS; 4) la burocracia de las instituciones de salud y otras dependencias de gobierno (específicamente para la atención de sobrevivientes de violencia sexual se identificó que a las mujeres migrantes las hacen esperar por mucho tiempo con la finalidad de desmotivar la denuncia, solicitan pruebas que muchas veces no pueden conseguir y testigos con los que no cuentan, aunado a actitudes discriminatorias y revictimizantes que dificultan el acceso a la justicia); 5) la falta de personal de salud capacitado técnicamente en los hospitales (ej. especialistas que sepan cómo operar el ultrasonido); 6) el desconocimiento sobre los protocolos a seguir con las mujeres sobrevivientes de violencia sexual, pues se identificaron casos en los que no se indica anticoncepción de emergencia, sobre todo con adolescentes migrantes; 7) el desconocimiento del contexto en el migran las mujeres, lo cual trae consigo una falta de sensibilización del personal en las unidades de salud; 8) la negación de servicios, debido a que las mujeres migrantes en muchas ocasiones no cuentan con los documentos solicitados para que se les brinde la atención; 9) la discriminación del personal hacia las mujeres que son sobrevivientes de violencia sexual, sobre todo con adolescentes migrantes embarazadas; 10) la presencia de violencia obstétrica; 11) el miedo del personal de salud 
de violencia obstétrica; 11) el miedo del personal de salud de proporcionar atención a la población migrante, ya que piensan que puede tener consecuencias legales hacia ellas y ellos si brindan la atención (esto fue mencionado también con relación a la interrupción del embarazo); y, 12) la presencia de objetores de conciencia, desaconsejando a las mujeres migrantes que recurran a la interrupción del embarazo, aunque se mencionó que en algunas ocasiones el personal médico se declara como objetor de conciencia pero por el miedo a tener un problema legal.

Con respecto a las organizaciones de la sociedad civil, las barreras que se identificaron fueron 1) la cobertura limitada, pues hay algunas organizaciones que únicamente brindan servicios a la población que llega al albergue, por lo que su capacidad para brindar atención a las personas migrantes es limitada; 2) dificultades económicas que se reflejan en la falta de recursos y desabasto de medicamentos ya que son recurrentes los casos en los que las OSCs no pueden cubrir los gastos relacionados con la atención de las mujeres migrantes (ej. comprar medicinas y realizar ultrasonidos); y 3 ) en el caso de organizaciones religiosas que trabajan con población migrante, sus creencias pueden llegar a intervenir con el acceso a ciertos servicios de SSyR, sobre todo relacionados con anticoncepción de emergencia y la interrupción del embarazo.

Por último, las barreras individuales identificadas fueron 1) la constante movilidad de las mujeres migrantes, ya que no se detienen mucho tiempo en un solo lugar, lo cual dificulta el acceso a información y su seguimiento (ej. mujeres que no quieren interrumpir su viaje para tomar el kit de violencia sexual), a excepción de la población que pasa más tiempo en los albergues, donde su acceso a servicios es mayor en comparación con quienes permanecen pocos días; 2) el factor económico por la dificultad para pagar estudios, consultas y medicamentos -como los ultrasonidos o el ácido fólico que no se proporciona de forma gratuita para mujeres

Tabla 2. .. Resumen de principales barreras para que las mujeres migrantes accedan a los servicios de salud en Tabasco, 2020.

\begin{tabular}{|c|c|}
\hline Clasificación & Principales barreras \\
\hline Normativas & -No se identificaron \\
\hline Institucionales & $\begin{array}{l}\text {-Infraestructura } \\
\text {-Tiempo de espera } \\
\text {-Traslados } \\
\text {-Desabasto de medicamentos e insumos médicos } \\
\text {-Burocracia } \\
\text {-Falta de personal capacitado } \\
\text {-Desconocimiento de los protocolos } \\
\text {-Desconocimiento del contexto en el que las mujeres migran } \\
\text {-Falta de sensibilización } \\
\text {-Negación de servicios } \\
\text {-Discriminación } \\
\text {-Violencia obstétrica } \\
\text {-Miedo del personal de salud y presencia de objetores de conciencia }\end{array}$ \\
\hline Organizacionales & $\begin{array}{l}\text {-Cobertura } \\
\text {-Falta de recursos y desabasto de medicamentos } \\
\text {-Creencias religiosas }\end{array}$ \\
\hline Individuales & $\begin{array}{l}\text {-Movilidad } \\
\text {-Dificultad económica } \\
\text {-Violencia de género } \\
\text {-Normalización de la violencia } \\
\text {-Falta de autocuidado } \\
\text {-Desconocimiento } \\
\text {-Miedo } \\
\text {-Tabús, mitos y estigma relacionados con la SSyR }\end{array}$ \\
\hline
\end{tabular}


embarazadas-, por lo que las mujeres buscan un apoyo económico para acceder a esos servicios y, en algunos casos recurren a la prostitución por falta de un trabajo en el que las acepten; 3) la presencia de violencia de género hacia las mujeres migrantes por parte de sus parejas afectivas, sean éstas de largo tiempo o conocidas en el transcurso del viaje $\neg$-el acceso a los servicios de SSyR se dificulta si la mujer viaja con el agresor- - , o la fuerte discriminación hacia las mujeres lesbianas en las "caravanas", donde se les insulta, ofende y violenta por su orientación sexual; 4) la normalización de la violencia, en el caso concreto de las mujeres migrantes, algunas consideran que al acceder a ciertos intercambios sexuales no se denomina violencia sexual o violencia de género y no lo nombran ni reconocen como tal; 5) la falta de autocuidado asociada a que la preservación de la vida y la continuidad del viaje prevalece sobre la salud, lo cual se refleja en que las mujeres embarazadas no acceden a consultas prenatales y servicios de salud antes del parto, y a partir de la pandemia de COVID-19, se observa que las mujeres no usan cubrebocas, aunque se les pide constantemente; 6) el desconocimiento sobre sus derechos, aspectos legales y dónde pueden recibir la atención que necesitan, así como sobre la anticoncepción de emergencia (cuándo, dosis, vía de administración); 7) la presencia de miedo, tanto de acudir a los servicios y ser identificadas y deportadas, como de acceder a servicios de interrupción del embarazo, ya que muchas de estas mujeres provienen de países donde el aborto es penalizado y piensan que las leyes de su país son iguales a las de México; y, 8) existen muchos tabús en torno a la sexualidad, lo que dificulta que las mujeres hablen acerca de esos temas y que, consecuentemente, acudan a atención.

Cuando la población migrante no encuentra otra salida para sobrevivir o hacer frente a algún problema, una estrategia que tienen es desistir y entregarse a las autoridades migratorias en México.

\section{COVID-19, mujeres migrantes y acceso a servicios de SSYR}

En general, y de acuerdo con el reporte de las organizaciones entrevistadas en los siete estados que son parte de este estudio $^{1}$, se identificó que la pandemia por COVID-19 tuvo los siguientes efectos en las OSCs y en la población migrante, particularmente en las mujeres y su acceso a servicios de SSyR:
- Reducción de unidades de salud y servicios disponibles. La reconversión de clínicas y hospitales a Centros COVID y la atención restringida a casos de emergencia, limitó de manera significativa la atención en las unidades médicas, ya sea por retraso o negación de la atención.

- Cierre temporal y/o parcial de albergues. Incluso aquellos que se mantuvieron abiertos dejaron de recibir a personas, ya que las y los migrantes no acudían porque escucharon que estaban cerrados.

- Disminución de recursos en las OSCs para prestar la atención. Con la pandemia hubo una reducción de recursos recibidos a través de donativos, además de tener una baja en el número de personas voluntarias, y del personal en general para brindar servicios y atención, incluyendo a personal médico.

- Permanencia prolongada de migrantes en los lugares, al no poder seguir su viaje. El cierre de oficinas gubernamentales -como la Comar- afectó a aquellas personas que esperaban la resolución a su solicitud de condición de refugiada u otro trámite similar, y les obligó a no salir de la entidad donde iniciaron el procedimiento.

- Afectaciones a la salud mental de las personas migrantes. Incremento de las preocupaciones relacionadas con sus procesos frente a autoridades migratorias, y/o por el confinamiento en albergues.

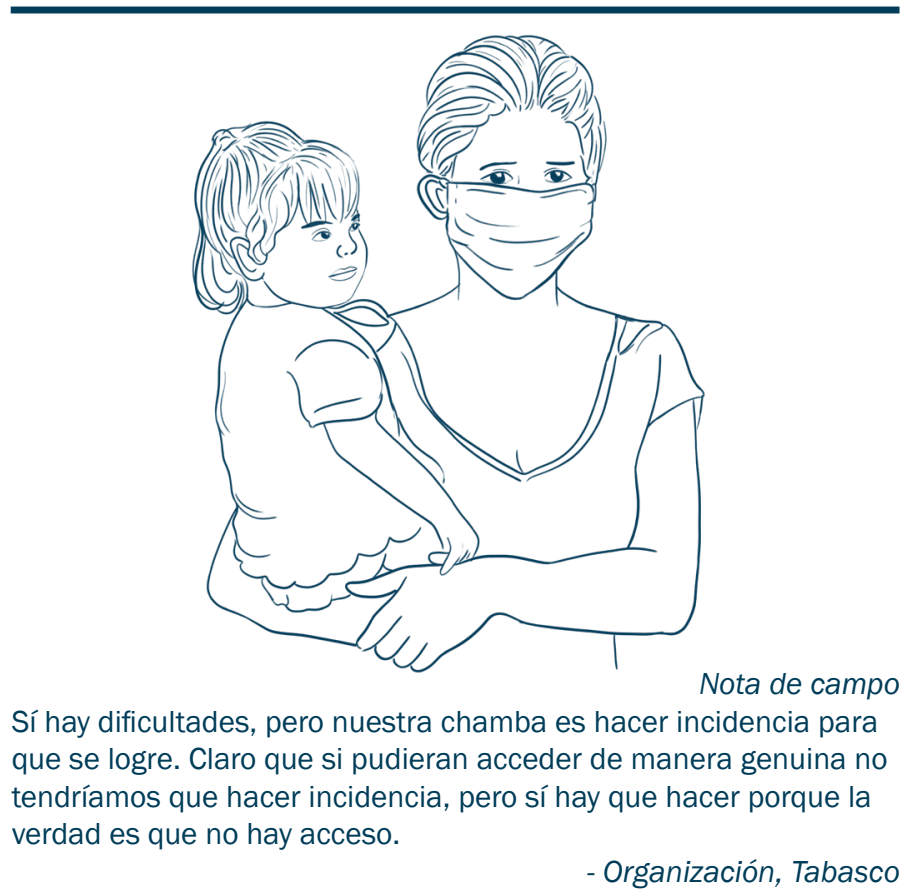

- Organización, Tabasco 
- Pérdida de trabajos y recursos económicos. Con el cierre de establecimientos comerciales hubo pérdida de empleos que también afectó a personas migrantes, pues se enfentan a la imposibilidad de laborar y tener el ingreso para solventar sus necesidades básicas.

- Miedo a asistir a las unidades médicas. Las personas migrantes tienen temor a contagiarse si asisten a las unidades médicas, por lo que retrasan o desisten de la atención.

\section{CONCLUSIONES Y ÁREAS DE OPORTUNIDAD ${ }^{2}$}

Las posibilidades para mejorar la atención de la salud sexual y reproductiva de las mujeres migrantes son amplias, desde el fortalecimiento del sistema de salud público que garantice el acceso a estos servicios, hasta apoyando a las organizaciones y albergues que están dando o facilitando el acceso.

Las organizaciones están dedicando sus recursos a garantizar el acceso a los servicios donde observan que hay vacíos en la atención, o una atención insuficiente, permitiendo el cumplimiento de lo que es un derecho humano. Si bien las osCs no son responsables garantes de brindar servicios de salud, ha demostrado ser clave para contribuir a este propósito. En el campo en que se enfocó el estudio (SSyR de las mujeres migrantes), el rol de las organizaciones de la sociedad civil es particularmente relevante, no sólo por ser muchas veces el primer punto de contacto, sino porque la confianza que ganan con su trabajo les habilita para tratar estos temas -considerados de mayor intimidad-.

El Estado, a través de sus instituciones, tiene la responsabilidad de garantizar que los servicios de salud se brinden de forma oportuna a las mujeres migrantes. El apoyo a la continua capacitación de servidores públicos sobre los derechos de personas migrantes en México (y sobre lo que implican los cambios en políticas públicas) puede fortalecer la viabilidad de las rutas de acceso a servicios de salud.

Por su parte, en las instituciones de salud se identificaron varias barreras para la atención, muchas de ellas relacionadas con problemáticas estructurales (ej. infraestructura insuficiente y lejana, falta de insumos, capacitación técnica de personal de salud), pero también vinculadas con la insuficiente sensibilización y conocimiento de protocolos para la atención a la población migrante. La prestación de servicios se supedita en ocasiones a la presentación de documentos que las mujeres no traen consigo o no tienen. La Secretaría de Salud, a través de las jurisdicciones sanitarias, tiene la responsabilidad de garantizar que los servicios de SSyR (incluida la interrupción del embarazo por las causales legales en el estado) se brinden de forma oportuna a las mujeres migrantes, y de llevar a cabo las tareas necesarias (ej. capacitar y sensibilizar al personal que labora en sus instituciones) para que la atención sea de la más alta calidad posible.

Con base en el reporte de las organizaciones en Tabasco se identificaron áreas de oportunidad y responsabilidad de la Secretaría de Salud, como incidir políticamente para lograr financiamiento suficiente y que responda a las necesidades de salud de la población -incluida la suficiencia de insumos como una amplia gama de métodos anticonceptivos-; mejorar el sistema de referencia a unidades de segundo nivel y facilitar la posibilidad de acceder a ellos; aumentar la contratación de personal médico capacitado y especialista (incluido personal capacitado para la interrupción del embarazo por los métodos recomendados por la Organización Mundial de la Salud). Capacitar sobre las obligaciones y protocolos operativos para la atención a la salud de las personas migrantes; y sensibilización a través de dinámicas efectivas para romper prejuicios hacia la población migrante. Promover un enfoque sensible y con perspectiva de género a la atención para la población en situación de movilidad entre el personal de las unidades de salud e incluir al personal médico y nomédico. Esto es particularmente importante para la atención a mujeres que vivieron violencia sexual. Capacitar al personal de salud sobre el aborto legal en el estado, y sobre la objeción de conciencia -derechos y responsabilidades-. Finalmente, la promoción de rendición de cuentas es importante para monitorear y evitar la negación de servicios a las mujeres migrantes, incluso si son indocumentadas o no cuentan con documentos probatorios de identidad.

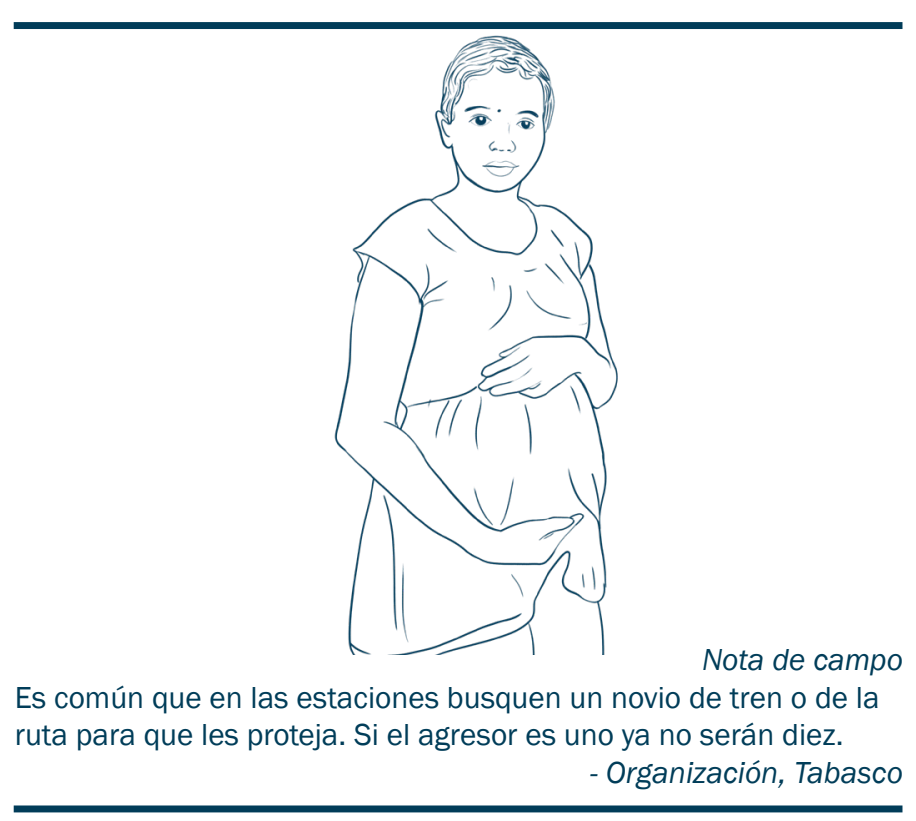


Tabla 3. Elementos a fortalecer en centros de salud y hospitales específicos. Tabasco, 2020.

\begin{tabular}{|c|c|c|}
\hline Unidad de salud & Municipio & Puntos a fortalecer \\
\hline Hospital Comunitario & Tenosique & $\begin{array}{l}\text { Falta de ultrasonidos, medicamentos e insumos. A su vez, } \\
\text { la rotación de personal administrativo es un reto para la } \\
\text { continuidad de las actividades y programas } \\
\text { implementados en el hospital. }\end{array}$ \\
\hline Hospital de la Mujer & Tenosique & $\begin{array}{l}\text { Es necesario brindar información a población migrante } \\
\text { sobre la atención médica, los procedimientos, etc. Dicha } \\
\text { información podría ser transmitida a través de folletos. }\end{array}$ \\
\hline $\begin{array}{l}\text { Hospital Regional Juan } \\
\text { Graham Casasus }\end{array}$ & Villahermosa & $\begin{array}{l}\text { Es necesario brindar información acerca del área a donde } \\
\text { se podrían dirigir las mujeres migrantes, así como } \\
\text { mejorar el trato del personal hacia la población migrante. }\end{array}$ \\
\hline
\end{tabular}

En Tabasco, las organizaciones identificaron elementos a fortalecer en centros de salud y hospitales específicos:

En comparación con otros estados, las organizaciones que trabajan en Tabasco brindan, de forma directa o indirecta, métodos anticonceptivos, información y tratamiento de ITS, y atención a la salud menstrual. La atención integral a mujeres que han vivido violencia sexual es prioritaria para las OSCs, dada la necesidad que observan. En este sentido, Ilama la atención que la anticoncepción de emergencia y la interrupción del embarazo son servicios poco brindados (esto en línea con lo que ocurre en otras entidades). La capacidad de responder a las necesidades de las mujeres se hace mediante el trabajo en articulación, siendo la canalización y referencia entre ellas o con las unidades de salud el recurso más utilizado.

Sin embargo, las mismas organizaciones entrevistadas son conscientes de que no estar trabajando, o no lo suficiente, el tema de SSyR de las mujeres migrantes. La mitad de estas OSCs hicieron referencia a su interés por fortalecer la atención en SSyR a través de capacitaciones en género para ellas mismas, la generación de talleres sobre salud en general e incluir SSyR, o hacer sesiones más regulares en los casos que ya tienen, y diseñar estrategias para trabajar específicamente estos temas con sociedad civil, contrapartes u otras organizaciones e instituciones de salud.

Al interior de las organizaciones en Tabasco (tanto albergues, como organizaciones nacionales e internacionales) se i dentificó al recurso económicos como la principal limitante para prestar y facilitar la atención en SSyR. La dificultad financiera las limita a ofrecer servicios a las personas que se acercan a ellas, e incluso les impide a cubrir los gastos que el sistema público de salud obliga a recurrir en la atención. Un obstáculo de naturaleza ideológica es la característica religiosa de algunos albergues, que reducen su capacidad para brindar información sobre la pastilla de emergencia y la interrupción del embarazo de acuerdo con la normativa permitida en el estado.

En Villahermosa las organizaciones se consideran fuera de la ruta con mayor flujo de migrantes y son conscientes de que si estuvieran más cerca de Tenosique podría ser mayor su trabajo con esta población y con otras instancias. Por ejemplo, trabajar con la oficina de ACNUR implica muchas veces desplazarse a este lugar. Aun así, señalaron la posibilidad de generar en ese municipio una mesa de trabajo interinstitucional y transdisciplinaria para abordar el tema de migración y SSyR. Además, otra acción que plantearon fue hacer un grupo de médicas y médicos no objetores de conciencia, que conozcan bien las leyes y protocolos, y que puedan realizar interrupciones de embarazos.

Algunas de las organizaciones entrevistadas consideraron de suma importancia establecer vinculación, redes de apoyo y capacitaciones entre todas las OSCs que trabajan con población migrante, lo cual permitirá fortalecer el conocimiento y de alguna forma favorecer el acceso a los 
servicios de SSyR. También mencionaron la importancia de realizar actividades de abogacía con autoridades estatales para visibilizar la problemática a la que están expuestas las mujeres migrantes en relación con su SSyR. Para ello se considera necesario contar con una base de datos con información sobre las problemáticas de salud de las mujeres migrantes, ya que a la fecha coinciden en que no existe registro de esta información.

En resumen, las organizaciones de la sociedad civil pueden, entre otras cosas, ayudar a monitorear las necesidades de SSyR de esta población; brindar información/educación de calidad sobre la SSyR (con base en las necesidades identificadas), y sobre los pasos para ejercer su derecho a la salud; identificar (y educar también sobre) síntomas y señales de riesgo que indican que una mujer debe de ser atendida de urgencia; y establecer mecanismos formales de referencia a unidades de salud correspondientes con el tema a tratar.

Otra área de oportunidad es la de contar con un sistema de información que les permita el monitoreo de la población a la que atienden y los problemas de salud (en general y en particular, SSyR) presentados $\neg$-entre otras temáticas relacionadas con el respeto a sus derechos humanos-. La finalidad del monitoreo es la de sistematizar e identificar las necesidades más apremiantes de esta población y cómo se van transformando en el tiempo. Esto ayudará a las organizaciones a contar con información para hacer incidencia con las instituciones de salud gubernamentales, y recomendar la priorización de esfuerzos y recursos en el corto, mediano, y largo plazo. El sistema de información se puede generar también con el apoyo de organizaciones de la sociedad civil nacionales y/o internacionales. Con el fin de hacerse de recursos para la creación de este sistema de información seguro y compartido entre las redes de apoyo a migrantes, se puede insistir a través de la incidencia con quienes financian trabajo en este campo.

Todas las organizaciones de la sociedad civil hacen lo que está en sus manos para cubrir las necesidades de salud de las mujeres migrantes, incluidas las de salud. En el corto plazo, el apoyo gubernamental estatal y federal, de agencias internacionales, y de personas individuales a estas organizaciones de la sociedad civil es también necesario. Por tanto, se hace un Ilamado a estos actores a no desestimar el papel que las OSCs cubren para procurar el bienestar de las personas migrantes, y en consecuencia, financiar su trabajo. A pesar de las limitaciones del estudio (como contar sólo con el reporte de las OSCs, y no con la perspectiva ni de las mujeres ni del sistema de salud local), la investigación muestra importantes áreas de oportunidad para lograr la atención a la SSyR de las mujeres migrantes. Investigaciones futuras deberán de indagar directamente sobre las necesidades de SSyR de mujeres, adolescentes y niñas, y las necesidades del sector salud para poder prestar la atención. La investigación operativa puede mejorar el funcionamiento de las OSCs como puentes para acercar a las mujeres a los servicios de salud, y apoyar a las unidades médicas a mejorar sus protocolos, así como a entender por qué ciertos esfuerzos - de capacitación, por ejemplo-, pueden no estar dando el resultado esperado.

La salud sexual y reproductiva de las mujeres migrantes en tránsito por México es un derecho. Tomar conciencia de que las necesidades de salud de las mujeres son minimizadas, incluso por ellas mismas, es uno de los retos hacia la equidad de género. Facilitar y garantizar el cuidado a su salud forma parte de los esfuerzos para lograr el respeto a los derechos humanos.

Agradecemos el esfuerzo realizado por el equipo de investigación durante los meses de trabajo, así como la gran disposición que las personas entrevistadas tuvieron para compartirnos su experiencia en medio de la pandemia de SARS-CoV-2, y por su compromiso constante con las mujeres migrantes.

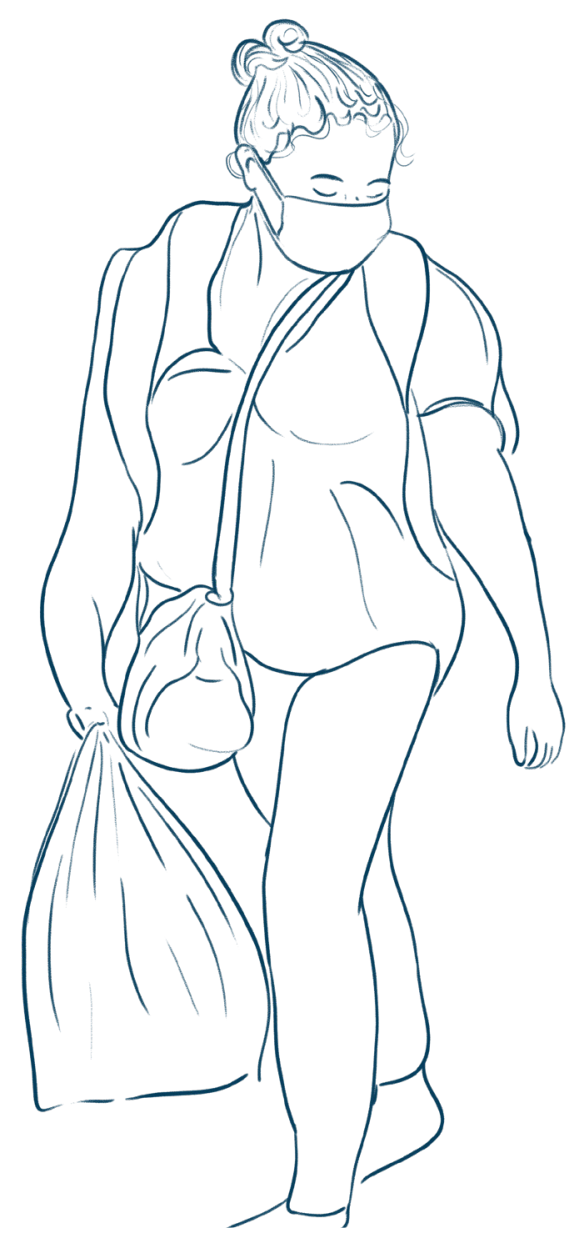


El presente estudio es evidencia de que las organizaciones de la sociedad civil locales e internacionales trabajan en primera línea para defender, promover, y apoyar el alcance de los derechos de las personas migrantes -incluso coadyuvando en el acceso a servicios de salud sexual y reproductiva-.

El reconocimiento a ellas y a su labor va acompañado de un llamado a la solidaridad para impulsar su financiamiento y generar condiciones que permitan su desarrollo y el de sus colaboradoras/es.

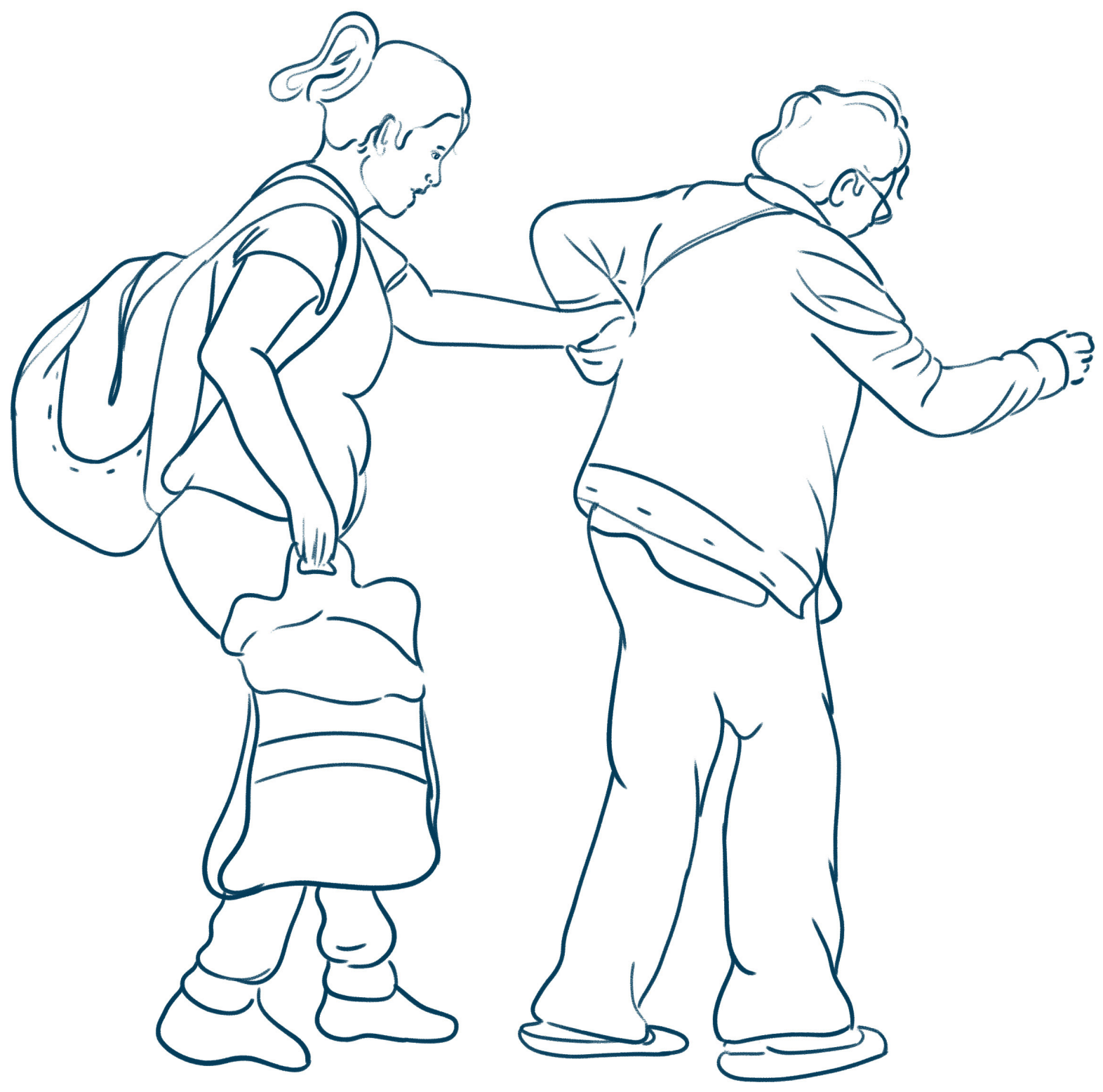

Population Council México. 2021. Atención de la salud sexual y reproductiva de mujeres migrantes: Un mapeo de actores de la sociedad civil en México. Resultados principales para el estado de Chiapas. Ciudad de México, México: Population Council. 
REFERENCIAS:

1. ACNUR. Encuesta sobre la Población Refugiada en México. México, 2017. Disponible en: https://www.acnur.org/publications/pub_inf/5ddff2ca4/encuesta-sobre-la-poblacion-refugiada-en-mexico-enpore-2017.html

2. Alianza Nacional por el Derecho a Decidir (Andar). Aborto Legal. Baja California. México, 2020. Disponible en: https://andar. org.mx/aborto-legal/\#BAJACALIFORNIA

3. Red de Documentación de las Organizaciones Defensoras de Migrantes (REDODEM), Procesos migratorios en México, nuevos rostros, nuevas dinámicas. Informe 2018. México, 2019.

4. Unidad de Política Migratoria, Registro e Identidad de las Personas (UPMRIP). Estadísticas Migratorias. Síntesis 2020. Secretaría de Gobernación. México, 2020. 